\title{
Graphs Whose Minimal Rank is Two: The Finite Fields Case
}

\author{
Wayne Barrett \\ wayne@math.byu.edu \\ Hein Van Der Holst \\ Raphael Loewy
}

Follow this and additional works at: https://scholarsarchive.byu.edu/facpub

Part of the Mathematics Commons

\section{Original Publication Citation}

Barrett, Wanye, Van Der Holst, Hein, and Loewy, Raphael, Graphs Whose Minimal Rank is Two:

The Finite Fields case, Elec. Journal of Linear Algebra (25): Vol. 14 p. 32-42.

\section{BYU ScholarsArchive Citation}

Barrett, Wayne; Van Der Holst, Hein; and Loewy, Raphael, "Graphs Whose Minimal Rank is Two: The Finite Fields Case" (2005). Faculty Publications. 395.

https://scholarsarchive.byu.edu/facpub/395

This Peer-Reviewed Article is brought to you for free and open access by BYU ScholarsArchive. It has been accepted for inclusion in Faculty Publications by an authorized administrator of BYU ScholarsArchive. For more information, please contact ellen_amatangelo@byu.edu. 


\title{
GRAPHS WHOSE MINIMAL RANK IS TWO: THE FINITE FIELDS $\mathrm{CASE}^{*}$
}

\author{
WAYNE BARRETT ${ }^{\dagger}$, HEIN VAN DER HOLST ${ }^{\ddagger}$, AND RAPHAEL LOEWY ${ }^{\S}$
}

\begin{abstract}
Let $F$ be a finite field, $G=(V, E)$ be an undirected graph on $n$ vertices, and let $S(F, G)$ be the set of all symmetric $n \times n$ matrices over $F$ whose nonzero off-diagonal entries occur in exactly the positions corresponding to the edges of $G$. Let $\operatorname{mr}(F, G)$ be the minimum rank of all matrices in $S(F, G)$. If $F$ is a finite field with $p^{t}$ elements, $p \neq 2$, it is shown that $\operatorname{mr}(F, G) \leq 2$ if and only if the complement of $G$ is the join of a complete graph with either the union of at most $\left(p^{t}+1\right) / 2$ nonempty complete bipartite graphs or the union of at most two nonempty complete graphs and of at most $\left(p^{t}-1\right) / 2$ nonempty complete bipartite graphs. These graphs are also characterized as those for which 9 specific graphs do not occur as induced subgraphs. If $F$ is a finite field with $2^{t}$ elements, then $\operatorname{mr}(F, G) \leq 2$ if and only if the complement of $G$ is the join of a complete graph with either the union of at most $2^{t}+1$ nonempty complete graphs or the union of at most one nonempty complete graph and of at most $2^{t-1}$ nonempty complete bipartite graphs. A list of subgraphs that do not occur as induced subgraphs is provided for this case as well.
\end{abstract}

Key words. Rank 2, Minimum rank, Symmetric matrix, Forbidden subgraph, Bilinear symmetric form, Finite field.

AMS subject classifications. 05C50, 05C75, 15A03, $15 \mathrm{~A} 57$.

1. Introduction. Let $F$ be a field. For any graph $G=(V, E)$ with $V=$ $\{1,2, \ldots, n\}$ (all graphs in this paper are considered undirected and simple), let $S(F, G)$ be the set of all symmetric $n \times n$ matrices $A=\left(a_{i, j}\right)$ with entries in $F$ such that $a_{i, j} \neq 0, i \neq j$, if and only if $i j \in E$. There is no restriction on the main diagonal entries of $A$. Let

$$
\operatorname{mr}(F, G)=\min \{\operatorname{rank} A \mid A \in S(F, G)\}
$$

In this paper we identify, for any finite field $F$, those graphs $G$ such that $\operatorname{mr}(F, G) \leq 2$. This has been done in [1] for the case that $F$ is an infinite field. We will use several results from that paper.

We will use several concepts from graph theory. The complement of a graph $G=(V, E)$ is the graph $G^{c}=\left(V, E^{c}\right)$. If $G_{1}=\left(V_{1}, E_{1}\right)$ and $G_{2}=\left(V_{2}, E_{2}\right)$ are two graphs, the union of $G_{1}$ and $G_{2}$ is the graph $G_{1} \cup G_{2}=\left(V_{1} \cup V_{2}, E_{1} \cup E_{2}\right)$. We abbreviate $G \cup G \cup \cdots \cup G$ ( $m$ times) to $m G$. The join of $G_{1}$ and $G_{2}$ is the graph obtained from $G_{1} \cup G_{2}$ by adding an edge between each vertex of $G_{1}$ and each vertex

${ }^{*}$ Received by the editors 22 November 2004. Accepted for publication 13 January 2005. Handling Editor: Richard A. Brualdi.

${ }^{\dagger}$ Department of Mathematics, Brigham Young University, Provo, UT 84602, USA (wayne@math.byu.edu).

$¥$ Faculty of Mathematics and Computer Science, Eindhoven University of Technology, $5600 \mathrm{MB}$ Eindhoven, The Netherlands (Hein.van.der.Holst@cwi.nl).

$\S$ Department of Mathematics, Technion-Israel Institute of Technology, Haifa 32000, Israel (loewy@techunix.technion.ac.il). The research of this author was supported by the M. and M. Bank Mathematics Research Fund. 
of $G_{2}$. We denote the join of $G_{1}$ and $G_{2}$ by $G_{1} \vee G_{2}$. So $G_{1} \vee G_{2}=\left(G_{1}^{c} \cup G_{2}^{c}\right)^{c}$. A vertex $u$ of $G$ is a dominating vertex if $u$ is adjacent to all other vertices of $G$.

We denote the path on $n$ vertices by $P_{n}$. The complete graph on $n$ vertices will be denoted by $K_{n}$ and by a triangle we mean $K_{3}$. The complete bipartite graph $K_{m, n}$ is the complement of $K_{m} \cup K_{n}$. We allow $m$ or $n$ to be equal to 0 , in which case $K_{m, 0}=m K_{1}$ and $K_{0, n}=n K_{1}$. The complete tripartite graph $K_{n_{1}, n_{2}, n_{3}}$ is the complement of $K_{n_{1}} \cup K_{n_{2}} \cup K_{n_{3}}$. Other graphs we will use are depicted in Figure 1.1. Notice that $\hat{W}_{4}=\left(K_{2} \cup P_{3}\right)^{c}$, paw $=\left(K_{1} \cup P_{3}\right)^{c}$, diamond $=\left(2 K_{1} \cup K_{2}\right)^{c}$, $\ltimes=\left(\text { diamond } \cup K_{1}\right)^{c}$, and dart $=\left(K_{1} \cup \text { paw }\right)^{c}$.

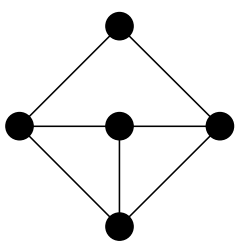

$\hat{W}_{4}$

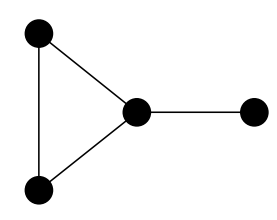

paw

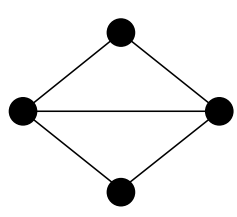

diamond

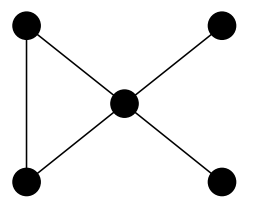

$\ltimes$

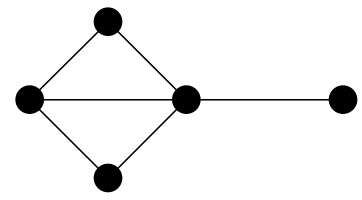

dart

FIG. 1.1. Some special graphs

If $F$ is a finite field with $p^{t}$ elements, $p \neq 2$, we show that $\operatorname{mr}(F, G) \leq 2$ if and only if the complement of $G$ is the join of a complete graph with either the union of at most $\left(p^{t}+1\right) / 2$ nonempty complete bipartite graphs or the union of at most two nonempty complete graphs and of at most $\left(p^{t}-1\right) / 2$ nonempty complete bipartite graphs. If $F$ is a finite field with $2^{t}$ elements, we show that $\operatorname{mr}(F, G) \leq 2$ if and only if the complement of $G$ is the join of a complete graph with either the union of at most $2^{t}+1$ nonempty complete graphs or the union of at most one nonempty complete graph and of at most $2^{t-1}$ nonempty complete bipartite graphs.

The class of graphs $G$ satisfying $\operatorname{mr}(F, G) \leq 2$ can also be described in terms of forbidden subgraphs. We say that a graph $G$ is $H$-free if $G$ does not contain $H$ as an induced subgraph. If $\mathcal{F}$ is a set of graphs, we say that $G$ is $\mathcal{F}$-free if $G$ is $H$-free for each $H \in \mathcal{F}$. For any field $F$, a graph $G$ satisfying $\operatorname{mr}(F, G) \leq 2$ is $\left\{P_{4}, \ltimes\right.$, dart, $\left.P_{3} \cup K_{2}, 3 K_{2}\right\}$-free. Furthermore, if $F$ has char $F \neq 2$, then $G$ is $\bar{K}_{3,3,3^{-}}$ free, and if $F$ has char $F=2$, then $G$ is $\left(P_{3} \cup 2 K_{3}\right)^{c}$-free. If $F$ is an infinite field with char $F \neq 2$, then $P_{4}, \ltimes$, dart, $P_{3} \cup K_{2}, 3 K_{2}, K_{3,3,3}$ is a complete list of forbidden subgraphs for the class of graphs $G$ with $\operatorname{mr}(F, G) \leq 2$. If $F$ is an infinite field with 
char $F=2$, then $P_{4}, \ltimes$, dart, $P_{3} \cup K_{2}, 3 K_{2},\left(P_{3} \cup 2 K_{3}\right)^{c}$ is such a list. See [1] for proofs of these claims. In this paper we will give the forbidden subgraphs for the case that $F$ is a finite field. We will see that if $F$ is a finite field with char $F \neq 2$, there are 3 more forbidden subgraphs. If $F$ is a finite field with char $F=2$, there are 4 more forbidden subgraphs. These additional forbidden subgraphs depend on the number of elements in $F$. However, if $F$ is the field with two elements, three of these forbidden subgraphs are redundant. In that case there are 7 forbidden subgraphs for graphs $G$ satisfying $\operatorname{mr}(F, G) \leq 2$. These are $P_{4}$, dart, $\ltimes, P_{3} \cup K_{2}, 3 K_{2},\left(2 K_{2} \cup 2 K_{1}\right)^{c}$, and $\left(P_{3} \cup 2 K_{1}\right)^{c}$.

As an illustration of the problem of identifying those graphs $G$ with $\operatorname{mr}(F, G) \leq 2$, we now take a closer look at two specific graphs, $\left(P_{3} \cup 2 K_{1}\right)^{c}$ and $\left(3 K_{2} \cup K_{1}\right)^{c}$. Let $F_{2}, F_{3}$ be the finite fields with two and three elements, respectively.

If $A \in S\left(F_{2},\left(P_{3} \cup 2 K_{1}\right)^{c}\right)$, then up to permutation similarity,

$$
A=\left[\begin{array}{ccccc}
d_{1} & 1 & 1 & 1 & 0 \\
1 & d_{2} & 1 & 1 & 0 \\
1 & 1 & d_{3} & 1 & 1 \\
1 & 1 & 1 & d_{4} & 1 \\
0 & 0 & 1 & 1 & d_{5}
\end{array}\right]
$$

Then $\operatorname{rank} A \geq \operatorname{rank} A[145 \mid 235]=\operatorname{rank}\left[\begin{array}{ccc}1 & 1 & 0 \\ 1 & 1 & 1 \\ 0 & 1 & d_{5}\end{array}\right]=3$. If $F \neq F_{2}$, choose $a \in F$ such that $a \neq 0$ and $a \neq-1$. Then

$$
\left[\begin{array}{lllll}
1 & 1 & 1 & 1 & 0 \\
1 & 1 & 1 & 1 & 0 \\
1 & 1 & 1 & 1 & 0 \\
1 & 1 & 1 & 1 & 0 \\
0 & 0 & 0 & 0 & 0
\end{array}\right]+\left[\begin{array}{lllll}
0 & 0 & 0 & 0 & 0 \\
0 & 0 & 0 & 0 & 0 \\
0 & 0 & a & a & a \\
0 & 0 & a & a & a \\
0 & 0 & a & a & a
\end{array}\right] \in S\left(F,\left(P_{3} \cup 2 K_{1}\right)^{c}\right)
$$

and has rank 2 .

Now consider $\left(3 K_{2} \cup K_{1}\right)^{c}$. Although it would be tedious to verify directly, it follows from the results in [1] and those later in this paper that $\operatorname{mr}\left(F_{2},\left(3 K_{2} \cup K_{1}\right)^{c}\right)=$ $\operatorname{mr}\left(F_{3},\left(3 K_{2} \cup K_{1}\right)^{c}\right)=3$, while $\operatorname{mr}\left(F,\left(3 K_{2} \cup K_{1}\right)^{c}\right)=2$ for all other fields $F$. Indeed, it follows from the results in this paper that there are infinitely many pairs of distinct fields $F, F^{\prime}$ for which there exists a graph $G$ such that $\operatorname{mr}(F, G) \neq \operatorname{mr}\left(F^{\prime}, G\right)$. Thus, given an arbitrary finite field $F$, a systematic approach is needed to classify those graphs $G$ for which $\operatorname{mr}(F, G) \leq 2$.

2. Graphs $G$ with $\operatorname{mr}(F, G) \leq 2$. We begin this section with an easy observation, which is also mentioned in [1].

Observation 1 Let $F$ be a field and let $G$ be a graph on $n$ vertices. Then $\operatorname{mr}(F, G) \leq$ 1 if and only if $G$ can be expressed as the union of a complete graph and an independent set of vertices.

For any nonnegative integer $n$, let $S_{n}(F)$ be the set of all symmetric $n \times n$ matrices with entries in $F$. 
Lemma 2 Let $A \in S_{n}(F)$ with rank two. Then there is an invertible $B \in S_{2}(F)$ such that $A=U^{t} B U$, where $U$ is a $2 \times n$ matrix.

A proof of this elementary lemma is given in [1].

Let $B \in S_{2}(F)$ be invertible. Then $B$ defines a nondegenerate, bilinear symmetric form by

$$
(x, y) \rightarrow x^{t} B y, \quad x, y \in F^{2}
$$

A line in $F^{2}$ is a one dimensional subspace of $F^{2}$. If $L$ is a line, we denote its orthogonal complement (relative to the given form) by $L^{\perp}$. We can now distinguish two types of lines. Those for which $L \neq L^{\perp}$ and those for which $L=L^{\perp}$. The latter ones are called isotropic lines.

We denote the nonzero elements of a field $F$ by $F^{*}$.

Theorem 3 Let $F$ be a field with $p^{t}$ elements, $p$ prime and $p \neq 2$, and let $G=(V, E)$ be a graph on $n$ vertices. Then, if $\operatorname{mr}(F, G) \leq 2, G^{c}$ is either of the form

$$
\left(K_{p_{1}, q_{1}} \cup K_{p_{2}, q_{2}} \cup \cdots \cup K_{p_{k}, q_{k}}\right) \vee K_{r}
$$

for some appropriate nonnegative integers $r, k, p_{1}, q_{1}, p_{2}, q_{2}, \ldots, p_{k}, q_{k}$ with $p_{i}+q_{i}>0$, $i=1,2, \ldots, k$ and with $k \leq\left(p^{t}+1\right) / 2$, or of the form

$$
\left(K_{s_{1}} \cup K_{s_{2}} \cup K_{p_{1}, q_{1}} \cup K_{p_{2}, q_{2}} \cup \cdots \cup K_{p_{k}, q_{k}}\right) \vee K_{r}
$$

for some appropriate nonnegative integers $r, s_{1}, s_{2}, k, p_{1}, q_{1}, p_{2}, q_{2}, \ldots, p_{k}, q_{k}$ with $p_{i}+q_{i}>0, i=1,2, \ldots, k$ and with $k \leq\left(p^{t}-1\right) / 2$.

Proof. If a graph $G$ satisfies $\operatorname{mr}(F, G) \leq 1$ then $G$ is of the form $K_{m} \cup K_{n-m}^{c}$ for some nonnegative $m \leq n$, which is a special case of (2.2). So we may assume $\operatorname{mr}(F, G)=2$. In this case we follow the proof of Theorem 1 in [1]. Let $A \in S(F, G)$ with $\operatorname{rank} A=2$. Then according to Lemma $2, A$ can be written as $U^{t} B U$, where $B$ is an invertible $2 \times 2$ symmetric matrix over $F$ and $U$ is a $2 \times n$ matrix over $F$.

Since char $F \neq 2$, we can assume that

$$
B=\left[\begin{array}{cc}
d_{1} & 0 \\
0 & d_{2}
\end{array}\right]
$$

for certain $d_{1}, d_{2} \in F^{*}$; see [2, pages 253-254]. A line $L$ spanned by the vector $x=\left[x_{1}, x_{2}\right]$ is isotropic if and only if $x_{1}^{2}+\left(d_{2} / d_{1}\right) x_{2}^{2}=0$. Hence isotropic lines exist in $F^{2}$ if and only if $-d_{2} / d_{1}$ is a square in $F$. Moreover, if $-d_{2} / d_{1}$ is a square in $F$, there are exactly two isotropic lines, those spanned by $\left[x_{1}, x_{2}\right]^{t}$ and $\left[x_{1},-x_{2}\right]^{t}$, where $x_{1}, x_{2} \in F^{*}$ satisfy $x_{1}^{2}+\left(d_{2} / d_{1}\right) x_{2}^{2}=0$.

Let $w_{i}$, for $i=1,2, \ldots, n$, be the $i$ th column of $U$. For any $i, j \in V$ with $i \neq j$ we have

$$
i j \in E\left(G^{c}\right) \leftrightarrow w_{i}^{t} B w_{j}=0
$$


Suppose that $r$ of the vectors $w_{1}, w_{2}, \ldots, w_{n}$ are 0 ; we may assume $w_{n-r+1}=w_{n-r+2}$ $=\cdots=w_{n}=0$. Then $G^{c}=H \vee K_{r}$. It remains to determine the structure of $H$.

Now, if $-d_{2} / d_{1}$ is not a square, there are no isotropic lines in $F^{2}$ and the number of pairs $\left(L, L^{\perp}\right)$ with $L$ not isotropic is $\left(p^{t}+1\right) / 2$. (The number of lines in $F^{2}$ is $p^{t}+1$ and the orthogonal complement of each line is distinct from the line itself.) For each pair $\left(L, L^{\perp}\right)$ with $L$ not isotropic, consider those $i, j \in V$ such that $\operatorname{Sp} w_{i}=L$ and $\operatorname{Sp} w_{j}=L^{\perp}$. These vertices induce a graph in $H$ which is either empty or a complete bipartite graph. Hence $H$ can be expressed as the union of at most $\left(p^{t}+1\right) / 2$ complete bipartite graphs, and so $G^{c}$ is of the form (2.1). If $-d_{2} / d_{1}$ is a square, there are two isotropic lines in $F^{2}$ and the number of pairs $\left(L, L^{\perp}\right)$ with $L$ not isotropic is $\left(p^{t}-1\right) / 2$. Denote the isotropic lines by $L_{1}$ and $L_{2}$. The vertices $i \in V$ such that $\operatorname{Sp} w_{i}=L_{1}$ induce a clique in $H$, and similarly the vertices $i \in V$ such that $\operatorname{Sp} w_{i}=L_{2}$ induce a clique. For each pair $\left(L, L^{\perp}\right)$ with $L$ not isotropic, consider those $i, j \in V$ such that $\operatorname{Sp} w_{i}=L$ and $\operatorname{Sp} w_{j}=L^{\perp}$. This induces a complete bipartite graph in $H$. Hence $H$ can be expressed as the union of at most 2 complete graphs and of at most $\left(p^{t}-1\right) / 2$ complete bipartite graphs, and so $G^{c}$ is of the form (2.2).

Theorem 4 Let $F$ be a field with $p^{t}$ elements, $p$ prime and $p \neq 2$, and let $G$ be a graph whose complement is of the form (2.1) or of the form (2.2). Then $m r(F, G) \leq 2$.

Proof. Let us first assume that $G$ has the form (2.1). Take an element $-d$ of $F$ which is not a square in $F$. Let $E=\left[\begin{array}{ll}1 & 0 \\ 0 & d\end{array}\right]$. It suffices to show there exists a $U \in F^{2, n}$ such that $A=U^{t} E U \in S(F, G)$. Since $-d$ is not a square, we know that no line in $F^{2}$ is isotropic with respect to the bilinear form defined by $E$. The number of pairs $\left(L, L^{\perp}\right)$ with $L$ not isotropic is $\left(p^{t}+1\right) / 2$. Take from each such pair one line, and denote them by $L_{1}, L_{2}, \ldots, L_{\left(p^{t}+1\right) / 2}$. Let $x^{(i)}$ be a vector spanning $L_{i}$ and let $y^{(i)}$ be a nonzero vector orthogonal to $x^{(i)}$. Let $w_{i}, i=1,2, \ldots, n$ denote the columns of $U$. Choose $w_{n-r+1}=w_{n-r+2}=\cdots=w_{n}=0$. Among $w_{1}, w_{2}, \ldots, w_{n-r}$ we pick the first $p_{1}$ equal to $x^{(1)}$ and the $q_{1}$ after these equal to $y^{(1)}$. Since $k \leq\left(p^{t}+1\right) / 2$, we can continue this process for all $p_{1}, q_{1}, p_{2}, q_{2}, \ldots, p_{k}, q_{k}$ and obtain a matrix $A$ of $\operatorname{rank} \leq 2$ in $S(F, G)$.

We now assume that $G$ has the form (2.2). Let

$$
E=\left[\begin{array}{rr}
1 & 0 \\
0 & -1
\end{array}\right]
$$

It suffices to show there exists a $U \in F^{2, n}$ such that $A=U^{t} E U \in S(F, G)$. This time there are two isotropic lines in $F^{2}$, namely the line spanned by $f_{1}=[1,1]^{t}$ and the line spanned by $f_{2}=[1,-1]^{t}$. The number of pairs $\left(L, L^{\perp}\right)$ with $L$ not isotropic, is $\left(p^{t}-1\right) / 2$. Take from each such pair one line, and denote them by $L_{1}, L_{2}, \ldots, L_{\left(p^{t}-1\right) / 2}$. Let $x^{(i)}$ be a vector spanning $L_{i}$ and let $y^{(i)}$ be a nonzero vector orthogonal to $x^{(i)}$. Let $w_{i}, i=1,2, \ldots, n$ denote the columns of $U$. Choose $w_{n-r+1}=w_{n-r+2}=\cdots=$ $w_{n}=0$. Choose $w_{1}, w_{2}, \ldots, w_{s_{1}}=f_{1}$ and choose $w_{s_{1}+1}, w_{s_{1}+2}, \ldots, w_{s_{1}+s_{2}}=f_{2}$. Among $w_{s_{1}+s_{2}+1}, w_{s_{1}+s_{2}+2}, \ldots, w_{n-r}$ we pick the first $p_{1}$ equal to $x^{(1)}$ and the $q_{1}$ 
after these equal to $y^{(1)}$. Since $k \leq\left(p^{t}-1\right) / 2$, we can continue this process for all $p_{1}, q_{1}, p_{2}, q_{2}, \ldots, p_{k}, q_{k}$ and obtain a matrix $A$ of $\operatorname{rank} \leq 2$ in $S(F, G)$.

If $F$ is a finite field with $\operatorname{char}(F)=2$, then each element is a square.

Theorem 5 Let $F$ be a field with $2^{t}$ elements and let $G$ be a graph on $n$ vertices. Then, if $\operatorname{mr}(F, G) \leq 2, G^{c}$ is either of the form

$$
\left(K_{s_{1}} \cup K_{s_{2}} \cup \cdots \cup K_{s_{k}}\right) \vee K_{r}
$$

for some appropriate nonnegative integers $k, r, s_{1}, s_{2}, \ldots, s_{k}$ with $k \leq 2^{t}+1$, or of the form

$$
\left(K_{s_{1}} \cup K_{p_{1}, q_{1}} \cup K_{p_{2}, q_{2}} \cup \cdots \cup K_{p_{k}, q_{k}}\right) \vee K_{r}
$$

for some appropriate nonnegative integers $k, r, s_{1}, p_{1}, q_{1}, p_{2}, q_{2}, \ldots, p_{k}, q_{k}$ with $k \leq 2^{t-1}$.

Proof. The same proof as the proof of Theorem 3 can be used here. Let $A \in$ $S(F, G)$ with rank 2 . Let $B=\left(b_{i, j}\right)$ be a $2 \times 2$ symmetric matrix and $U$ a $2 \times n$ matrix such that $A=U^{t} B U$. If $b_{1,1} \neq 0$ or $b_{2,2} \neq 0$, we can diagonalize $B$ by a congruence. So in this case we may assume

$$
B=\left[\begin{array}{cc}
d_{1} & 0 \\
0 & d_{2}
\end{array}\right]
$$

for certain $d_{1}, d_{2} \in F^{*}$. A line spanned by a nonzero vector $x=\left[x_{1}, x_{2}\right]^{t}$ is isotropic if and only if $d_{1} x_{1}^{2}+d_{2} x_{2}^{2}=0$. Since each element of $F$ is a square, there is an element $\beta \in F^{*}$ such that $\beta^{2}=-d_{2} / d_{1}$. Then $\left(x_{1}+\beta x_{2}\right)\left(x_{1}-\beta x_{2}\right)=0$; that is, there is exactly one isotropic line in $F$. If $b_{1,1}=0$ and $b_{2,2}=0$, we can assume

$$
B=\left[\begin{array}{ll}
0 & 1 \\
1 & 0
\end{array}\right] \text {. }
$$

In this case a line spanned by a nonzero vector $x=\left[x_{1}, x_{2}\right]^{t}$ is isotropic if and only if $x^{t} B x=x_{1} x_{2}+x_{2} x_{1}=0$. Hence every line is isotropic.

Now if each line in $F^{2}$ is isotropic, we are in case (2.3) and if there is one isotropic line in $F^{2}$, we are in case $(2.4)$.

Theorem 6 Let $F$ be a field with $2^{t}$ elements and let $G$ be a graph whose complement is of the form (2.3) or (2.4). Then $\operatorname{mr}(F, G) \leq 2$.

Proof. The proof is analogous to the proof of Theorem 4. In the case of (2.3) we take

$$
E=\left[\begin{array}{ll}
0 & 1 \\
1 & 0
\end{array}\right]
$$

and in the case of $(2.4)$ we take

$$
E=\left[\begin{array}{ll}
1 & 0 \\
0 & 1
\end{array}\right]
$$


3. Forbidden subgraphs. Let $F$ be a finite field. In the previous section we described the complements of the graphs whose symmetric matrices with entries in $F$ have minimum rank at most 2 . These graphs can also be described by means of forbidden subgraphs.

Proposition 7 [1] A graph $G$ can be expressed as the union of complete graphs and of complete bipartite graphs if and only if $G$ is $\left(P_{4}\right.$, paw, diamond)-free.

Proposition 8 A graph $G$ can be expressed as either the union of at most 2 complete graphs and of at most $m$ complete bipartite graphs or as the union of at most $m+1$ complete bipartite graphs if and only if $G$ is $\left(3 K_{3}, K_{2} \cup 2 K_{1} \cup m P_{3}, K_{1} \cup(m+1) P_{3}\right.$, $(m+2) K_{2} \cup K_{1}, P_{4}$, paw, diamond)-free.

Proof. $(\Rightarrow)$. From Proposition 7 it follows that $P_{4}$, paw, and diamond are forbidden subgraphs. If $G$ has $(m+2) K_{2}$ as an induced subgraph, then $G$ has no isolated vertices, and hence $G$ is $(m+2) K_{2} \cup K_{1}$-free. Since $G$ has at most two components which are complete graphs on 3 or more vertices, $3 K_{3}$ is a forbidden subgraph. If $G$ has $K_{2} \cup K_{1} \cup m P_{3}$ as an induced subgraph, then $G$ must be the union of two nonempty complete graphs and of $m$ nonempty complete bipartite graphs, and hence $G$ is $K_{2} \cup 2 K_{1} \cup m P_{3}$-free. If $G$ has $(m+1) P_{3}$ as an induced subgraph, then $G$ must be the union of $m+1$ nonempty complete bipartite graphs, and hence $G$ is $K_{1} \cup(m+1) P_{3}$-free.

$(\Leftarrow)$. By Proposition $7, G$ is of the form

$$
E_{1} \cup \cdots \cup E_{j} \cup H_{1} \cup \cdots \cup H_{l} \cup W, \quad 0 \leq j \leq 2,0 \leq l \leq m+1
$$

where the $E_{i}$ for $i=1, \ldots, j$ are complete graphs containing a triangle, the $H_{i}$ for $i=1,2, \ldots, l$ are complete bipartite graphs containing a $P_{3}$ as an induced subgraph, and $W$ is a collection of single edges and isolated vertices. Here we have used the assumption that $G$ is $3 K_{3}$-free to conclude that $j \leq 2$, and we have used the assumption that $G$ is $K_{1} \cup(m+1) P_{3}$-free to conclude that $l \leq m+1$. Since $G$ is $(m+2) K_{2} \cup K_{1}$-free, the number of single edges in $W$ is at most $m+2-j-l$.

We first assume that $j>0$. Then $l \leq m$ or else $K_{2} \cup 2 K_{1} \cup m P_{3}$ would be induced. If $W$ has $m+2-j-l$ single edges, then $W$ has no isolated vertices, and we may add $2-j$ single edges to the collection of complete graphs and $m-l$ single edges to the collection of complete bipartite graphs. Now suppose that $W$ has fewer than $2-j+m-l$ single edges and $l=m$ so that $j=1$. Then as $G$ is $K_{2} \cup 2 K_{1} \cup m P_{3^{-}}$ free, $W$ is either empty or contains exactly one vertex. If $W$ is nonempty, we add the vertex to the collection of complete graphs. It remains to consider the case in which $l<m$ and $W$ has $2-j+k$ single edges, where $k<m-l$. Add $2-j$ single edges to the collection of complete graphs, $k$ single edges to the collection of complete bipartite graphs and all the isolated vertices (as one bipartite graph) to the collection of complete bipartite graphs. In each case we have shown that $G$ can be expressed as the union of at most 2 complete graphs and of at most $m$ complete bipartite graphs.

Next assume that $j=0$. Then the number of single edges in $W$ is at most $m+2-l$. If $W$ has this number of single edges, then $W$ contains at least one single edge and $W$ 
has no isolated vertices. Since $G$ is $2 K_{1} \cup K_{2} \cup m P_{3}$-free, we know that $l \leq m$ in this case. We add 2 single edges to the collection of complete graphs and $m-l$ single edges to the collection of complete bipartite graphs. It remains to consider the case that the number of single edges in $W$ is at most $m+1-l$. If $l=m+1$, then $G$ can be expressed as the union of $m+1$ complete bipartite graphs, as $G$ is $K_{1} \cup(m+1) P_{3}$-free. If $l=m$ and $W$ contains one single edge, then $W$ contains at most one isolated vertex, as $G$ is $2 K_{1} \cup K_{2} \cup m P_{3}$-free. In this case we add the elements of $W$ to the collection of complete graphs. If $l=m$ and $W$ contains no single edge, then we add all the isolated vertices (as one bipartite graph) to the collection of complete bipartite graphs. Now suppose that $l<m$ and that $W$ contains $m+1-l$ single edges. We take 2 of the single edges to be complete graphs, each of the remaining $m-1-l$ single edges to be a complete bipartite graph, and all of the isolated vertices to be one complete bipartite graph. If $l<m$ and $W$ contains at most $m-l$ single edges, then we may regard each single edge as a complete bipartite graph, and all the isolated vertices to be one complete bipartite graph.

Theorem 9 [1] A graph $G$ has the form

$$
\left(K_{s_{1}} \cup K_{s_{2}} \cup \cdots \cup K_{s_{t}} \cup K_{p_{1}, q_{1}} \cup K_{p_{2}, q_{2}} \cup \cdots \cup K_{p_{k}, q_{k}}\right) \vee K_{r}
$$

for nonnegative integers $t, s_{1}, s_{2}, \ldots, s_{t}, k, p_{1}, q_{1}, p_{2}, q_{2}, \ldots, p_{k}, q_{k}, r$ with $p_{i}+q_{i}>0$, $i=1,2, \ldots, k$ if and only if $G$ is $\left(P_{4}\right.$, paw $\cup K_{1}$, diamond $\left.\cup K_{1}, \hat{W}_{4}, K_{2,2,2}\right)$-free.

Theorem 10 A graph $G$ is either of the form (2.1) or of the form (2.2) if and only if $G$ is $\left(P_{4}\right.$, paw $\cup K_{1}$, diamond $\cup K_{1}, \hat{W}_{4}, K_{2,2,2}, 3 K_{3},(m+2) K_{2} \cup K_{1}, K_{2} \cup 2 K_{1} \cup m P_{3}$, $\left.K_{1} \cup(m+1) P_{3}\right)$-free, where $m=\left(p^{t}-1\right) / 2$.

Proof. $(\Rightarrow)$. From Theorem 9 it follows that $P_{4}$, paw $\cup K_{1}$, diamond $\cup K_{1}, \hat{W}_{4}$, $K_{2,2,2}$ are forbidden subgraphs. Let

$$
\mathcal{F}=\left\{3 K_{3},(m+2) K_{2} \cup K_{1}, K_{2} \cup 2 K_{1} \cup m P_{3}, K_{1} \cup(m+1) P_{3}\right\},
$$

let $D$ be the set of dominating vertices of $G$, and let $C=V \backslash D$. Since no graph in $\mathcal{F}$ has a dominating vertex, a graph of $\mathcal{F}$ is induced in $G$ only if it is induced in $G[C]$. From Proposition 8 it follows that no graph of $\mathcal{F}$ is induced in $G[C]$.

$(\Leftarrow)$. By Theorem $9, G$ has the form (3.1). Let $D$ be the set of dominating vertices of $G$, and let $C=V \backslash D$. Then $G[C]$ can be expressed as the union of complete graphs and of complete bipartite graphs. So $G[C]$ is (paw, diamond)-free. Since $G[C]$ is $\left(P_{4}, 3 K_{3},(m+2) K_{2} \cup K_{1}, K_{2} \cup 2 K_{1} \cup m P_{3}, K_{1} \cup(m+1) P_{3}\right)$-free, it follows from Proposition 8 that $G[C]$ can be expressed as either the union of at most 2 complete graphs and of at most $m$ complete bipartite graphs or as the union of at most $m+1$ complete bipartite graphs. Hence, $G$ is either of the form (2.1) or of the form (2.2).

Theorem 11 Let $G$ be a graph and let $F$ be a finite field with $p^{t}$ elements, $p$ prime and $p \neq 2$. Then the following are equivalent: 
1. $m r(F, G) \leq 2$,

2. $G^{c}$ is either of the form (2.1) or of the form (2.2), and

3. $G$ is $\left(P_{4}\right.$, dart, $\ltimes, P_{3} \cup K_{2}, 3 K_{2}, K_{3,3,3},\left((m+2) K_{2} \cup K_{1}\right)^{c},\left(K_{2} \cup 2 K_{1} \cup m P_{3}\right)^{c}$, $\left.\left(K_{1} \cup(m+1) P_{3}\right)^{c}\right)$-free, where $m=\left(p^{t}-1\right) / 2$.

It is informative to compare Theorem 11 with the following result from [1].

Theorem 12 Let $G$ be a graph and let $F$ be an infinite field such that char $F \neq 2$. Then $m r(F, G) \leq 2$ if and only if $G$ is $\left(P_{4}, \ltimes\right.$, dart, $\left.P_{3} \cup K_{2}, 3 K_{2}, K_{3,3,3}\right)$-free.

Let $F$ be a finite field with $p^{t}$ elements, $p$ prime and $p \neq 2$, and let $m=\left(p^{t}-1\right) / 2$. With Theorem 11 and Theorem 12, we see that, if $\operatorname{mr}(\mathbb{R}, G) \leq 2$ and, additionally, $G$ is $\left(\left((m+2) K_{2} \cup K_{1}\right)^{c},\left(K_{2} \cup 2 K_{1} \cup m P_{3}\right)^{c},\left(K_{1} \cup(m+1) P_{3}\right)^{c}\right)$-free, then $\operatorname{mr}(F, G) \leq 2$.

Proposition 13 A graph $G$ can be expressed as either the union of at most $2^{t}+1$ complete graphs or as the union of at most one complete graph and of at most $2^{t-1}$ complete bipartite graphs if and only if $G$ is $\left(P_{3} \cup 2 K_{3}, P_{4}\right.$, paw, diamond, $\left.\left(2^{t-1}+1\right) K_{2} \cup\left(2^{t-1}+1\right) K_{1}, P_{3} \cup 2^{t-1} K_{2} \cup K_{1}, 2 K_{3} \cup 2^{t} K_{1}, 2^{t-1} P_{3} \cup 2 K_{1}\right)$-free

Proof. $(\Rightarrow)$. From Proposition 7 it follows that $P_{4}$, paw, diamond are forbidden subgraphs. If $G$ contains $P_{3}$ as an induced subgraph, it must be the union of at most one nonempty complete graph and of at most $2^{t-1}$ nonempty complete bipartite graphs. Hence $G$ is $P_{3} \cup 2 K_{3}$-free, $P_{3} \cup 2^{t-1} K_{2} \cup K_{1}$-free, and $2^{t-1} P_{3} \cup 2 K_{1}$-free. If $G$ contains $\left(2^{t-1}+1\right) K_{2} \cup K_{1}$ as an induced subgraph, then $G$ must be the union of at most $2^{t}+1$ nonempty complete graphs. Hence $G$ is $\left(2^{t-1}+1\right) K_{2} \cup\left(2^{t-1}+1\right) K_{1}$-free. If $G$ contains $2 K_{3}$ as an induced subgraph, $G$ must also be the union of at most $2^{t}+1$ nonempty complete graphs, and so $G$ must be $\left(2 K_{3} \cup 2^{t} K_{1}\right)$-free.

$(\Leftarrow)$. If $G$ is $P_{3}$-free, then $G$ is a union of complete graphs. We may express

$$
G=E_{1} \cup \cdots \cup E_{j} \cup W_{1} \cup \cdots \cup W_{k} \cup\left\{s_{1}, \ldots, s_{l}\right\},
$$

where the $E_{i}$ are the components containing a triangle, the $W_{i}$ are the single edges, and $\left\{s_{1}, \ldots, s_{l}\right\}$ is the collection of isolated vertices of $G$. If $j+k \geq 2^{t-1}+1$, then $G$ has at most $2^{t}+1-j-k$ isolated vertices, as $G$ is $\left(2^{t-1}+1\right) K_{2} \cup\left(2^{t-1}+1\right) K_{1}$-free. Hence $G$ can be expressed as the union of at most $2^{t}+1$ complete graphs in this case. If $j>1$, then $k+l \leq 2^{t}+1-j$, as $G$ is $2 K_{3} \cup 2^{t} K_{1}$-free. Hence, also in this case $G$ can be expressed as the union of at most $2^{t}+1$ complete graphs.

So we may assume $j+k \leq 2^{t-1}$ and $j \leq 1$. If $j=0$, then we add one single edge to the collection of complete graphs, we add the remaining single edges to the collection of complete bipartite graphs, and we add all the isolated vertices as one complete bipartite graph to the collection of complete bipartite graphs. If $j=1$, then $k \leq 2^{t-1}-1$. In this case we add all single edges to the collection of complete bipartite graphs, and we add all the isolated vertices as one complete bipartite graph to the collection of complete bipartite graphs. Hence we can express $G$ in each of these cases as the union of a complete graph and of at most $2^{t-1}$ complete bipartite graphs. 
So we may assume that $G$ has $P_{3}$ as an induced subgraph. By Proposition 7 , we may express

$$
G=E_{1} \cup \cdots \cup E_{j} \cup H_{1} \cup \cdots \cup H_{k} \cup W
$$

where the $E_{i}$ for $i=1,2, \ldots, j$ are complete graphs containing a triangle, the $H_{i}$ for $i=1,2, \ldots, k$ are complete bipartite graphs containing a $P_{3}$ as an induced subgraph, and $W$ is the collection of single edges and isolated vertices.

As $G$ has $P_{3}$ as an induced subgraph, $k \geq 1$. Since $G$ is $\left(P_{3} \cup 2 K_{3}\right)$-free, $0 \leq j \leq 1$. Since $G$ is $2^{t-1} P_{3} \cup 2 K_{1}$-free, $k \leq 2^{t-1}$, and if $k=2^{t-1}$, then $G$ has at most one other component besides the $H_{i}$ 's. So if $k=2^{t-1}$, we can express $G$ as the union of at most one complete graph and of $2^{t-1}$ complete bipartite graphs. We may therefore assume that $k<2^{t-1}$. Then, as $G$ is $\left(P_{3} \cup 2^{t-1} K_{2} \cup K_{1}\right)$-free, the number of single edges in $W$ is at most $2^{t-1}+1-k-j$ and if the number of single edges in $W$ is $2^{t-1}+1-k-j$, then $W$ has no isolated vertices.

If $W$ has $2^{t-1}+1-k-j$ single edges, then $2^{t-1}+1-k-j>0$, as we assumed $k<2^{t-1}$. So $W$ has at least one single edge. Hence $G$ can be expressed as the union of one complete graph and of $2^{t-1}$ complete bipartite graphs, where the complete graph is $E_{1}$ if $j=1$ and a single edge if $j=0$. If $W$ has $2^{t-1}-k-j$ single edges and $j=0$, then $W$ has at least one single edge, as $2^{t-1}-k>0$. Hence $G$ can be expressed as the union of one complete graph and of at most $2^{t-1}$ complete bipartite graphs, where the complete graph is one of the single edges and where all isolated vertices are put in one complete bipartite graph. If $W$ has $2^{t-1}-k-j$ single edges and $j=1$, then $G$ can be expressed as the union of one complete graph and of at most $2^{t-1}$ complete bipartite graphs, where the complete graph is $E_{1}$ and where all isolated vertices are put in one complete bipartite graph. If $W$ has fewer than $2^{t-1}-k-j$ single edges, then we add each single edge to the collection of complete bipartite graphs, and we add all the isolated vertices as one complete bipartite graph to the collection of complete bipartite graphs. Hence also in this case $G$ can be expressed as the union of at most one complete graph and of at most $2^{t-1}$ complete bipartite graphs.

Theorem 14 A graph $G$ is either of the form (2.3) or the form (2.4) if and only if $G$ is $\left(P_{4}\right.$, paw $\cup K_{1}$, diamond $\cup K_{1}, \hat{W}_{4}, K_{2,2,2}, P_{3} \cup 2 K_{3},\left(2^{t-1}+1\right) K_{2} \cup\left(2^{t-1}+1\right) K_{1}$, $\left.P_{3} \cup 2^{t-1} K_{2} \cup K_{1}, 2 K_{3} \cup 2^{t} K_{1}, 2^{t-1} P_{3} \cup 2 K_{1}\right)$-free.

We omit the proof since it is similar to the proof of Theorem 10.

Theorem 15 Let $G$ be a graph and let $F$ be a finite field with $2^{t}$ elements. Then the following are equivalent:

1. $\operatorname{mr}(F, G) \leq 2$,

2. $G^{c}$ is either of the form (2.3) or of the form (2.4), and

3. $G$ is $\left(P_{4}\right.$, dart, $\ltimes, P_{3} \cup K_{2}, 3 K_{2},\left(P_{3} \cup 2 K_{3}\right)^{c},\left(\left(2^{t-1}+1\right) K_{2} \cup\left(2^{t-1}+1\right) K_{1}\right)^{c}$, $\left.\left(P_{3} \cup 2^{t-1} K_{2} \cup K_{1}\right)^{c},\left(2 K_{3} \cup 2^{t} K_{1}\right)^{c},\left(2^{t-1} P_{3} \cup 2 K_{1}\right)^{c}\right)$-free.

The corresponding result proved in [1] for an infinite field with characteristic 2 is that $\operatorname{mr}(F, G) \leq 2$ if and only if the first 6 graphs in (3) are forbidden.

Specializing to the field $F_{2}$ with only two elements, we obtain 
Theorem 16 Let $G$ be a graph. Then $m r\left(F_{2}, G\right) \leq 2$ if and only if $G$ is $\left(P_{4}\right.$, dart, $\ltimes$, $\left.P_{3} \cup K_{2}, 3 K_{2}, P_{3} \vee P_{3},\left(P_{3} \cup 2 K_{1}\right)^{c}\right)$-free.

If we know that the graph $G$ is connected or, translated back to symmetric matrices, we know that the symmetric matrix is irreducible, then even fewer forbidden subgraphs characterize those graphs with minimum rank at most 2. For this we use the following corollary and proposition from [1].

Corollary 17 Let $G$ be connected. Then $G$ is $\left(P_{4}\right.$, dart, $\left.\ltimes, K_{3,3,3}\right)$-free if and only if $G$ is $\left(P_{4}, P_{3} \cup K_{1}, K_{2} \cup 2 K_{1}, K_{3,3,3}\right)$-free.

Proposition 18 Let $G$ be connected. Then $G$ is $\left(P_{4}\right.$, dart, $\left.\ltimes,\left(P_{3} \cup 2 K_{3}\right)^{c}\right)$-free if and only if $G$ is $\left(P_{4}, P_{3} \cup K_{1}, K_{2} \cup 2 K_{1},\left(P_{3} \cup 2 K_{3}\right)^{c}\right)$-free.

Theorem 19 Let $G$ be a connected graph and let $F$ be a finite field with $p^{t}$ elements, $p$ prime and $p \neq 2$. Then, $m r(F, G) \leq 2$ if and only if $G$ is $\left(P_{4}\right.$, dart, $\ltimes, K_{3,3,3}$, $\left.\left((m+2) K_{2} \cup K_{1}\right)^{c},\left(K_{2} \cup 2 K_{1} \cup m P_{3}\right)^{c},\left(K_{1} \cup(m+1) P_{3}\right)^{c}\right)$-free, where $m=\left(p^{t}-1\right) / 2$.

Proof. This follows from Theorem 11 and Corollary 17.

Theorem 20 Let $G$ be a connected graph and let $F$ be a finite field with $2^{t}$ elements. Then, $m r(F, G) \leq 2$ if and only if $G$ is $\left(P_{4}\right.$, dart, $\ltimes,\left(P_{3} \cup 2 K_{3}\right)^{c},\left(\left(2^{t-1}+1\right) K_{2} \cup\right.$ $\left.\left.\left(2^{t-1}+1\right) K_{1}\right)^{c},\left(P_{3} \cup 2^{t-1} K_{2} \cup K_{1}\right)^{c},\left(2 K_{3} \cup 2^{t} K_{1}\right)^{c},\left(2^{t-1} P_{3} \cup 2 K_{1}\right)^{c}\right)$-free.

Proof. In this case the theorem follows from Theorem 15 and Proposition 18.

Specializing to the field $F_{2}$ with only two elements, we get

Theorem 21 Let $G$ be a connected graph. Then $m r\left(F_{2}, G\right) \leq 2$ if and only $G$ is $\left(P_{4}\right.$, dart, $\left.\ltimes, P_{3} \vee P_{3},\left(P_{3} \cup 2 K_{1}\right)^{c}\right)$-free.

\section{REFERENCES}

[1] W. Barrett, H. van der Holst, and R. Loewy. Graphs whose minimal rank is two. Electron. J. of Linear Algebra, 11:258-280, 2004.

[2] P.M. Cohn. Basic algebra: groups, rings, and fields. Springer, London, 2002. 